\title{
Development of Thin-Film Composite Membranes for Nanofiltration at Extreme pH
}

\author{
Akbar Asadi Tashvigh,* Maria G. Elshof, and Nieck E. Benes* \\ Cite This: ACS Appl. Polym. Mater. 2021, 3, 5912-5919 \\ Read Online
}

ABSTRACT: Water recycling is one of the most sustainable Interfacial polymerization solutions to growing water scarcity challenges. However, wastewaters usually contain organic pollutants and often are at extreme $\mathrm{pH}$, which complicates the treatment of these streams with conventional membranes. In this work, we report the synthesis of a robust membrane material that can withstand prolonged exposure to extreme $\mathrm{pH}$ (of 1 or 13 for 2 months). Polyamine thin film composite (TFC) membranes are prepared in situ by interfacial polymerization between 1,3,5-tris(bromomethyl)benzene (tBrMeB) and $p$-phenylenediamine (PPD). Contrary to conventional polyamide TFC membranes, enhanced $\mathrm{pH}$ stability is achieved by eliminating the carbonyl groups from the polymer network. The membranes showed pure water


permeance and molecular weight cutoff (MWCO) of $0.28 \pm 0.09 \mathrm{~L} \mathrm{~m}^{-2} \mathrm{~h}^{-2} \mathrm{bar}^{-1}$ and $820 \pm 132 \mathrm{~g} \mathrm{~mol}^{-1}$, respectively. The membrane performance is further enhanced by manipulating the monomer structures and replacing $p$-phenylenediamine with $m$ phenylenediamine, resulting in a higher permeance of $1.3 \pm 0.3 \mathrm{~L} \mathrm{~m}^{-2} \mathrm{~h}^{-1} \mathrm{bar}^{-1}$ and a lower MWCO of $566 \pm 43 \mathrm{~g} \mathrm{~mol}^{-1}$. Given the ease of fabrication and excellent stability, this chemistry represents a step forward in the fabrication of robust membranes for industrial wastewater recycling.

KEYWORDS: $p H$ stability, polyamine, nanofiltration, interfacial polymerization, thin film composite membrane

\section{INTRODUCTION}

The freshwater shortage is undoubtedly one of the most disturbing issues of the current and future generations. It endangers the lives of millions of people around the globe. ${ }^{1}$ Additionally, the generation and discharge of industrial effluents into rivers and brackish water destroy the aquatic and wildlife, impacting human life directly. ${ }^{2,3}$ Therefore, a practical solution to eliminate the toxic compounds in the wastewater for reuse purposes would be highly beneficial.

Membrane-based separation technologies have proven their unique potential for the treatment of industrial wastewater and seawater desalination, which make them one of the sustainable solutions for the current water crisis. ${ }^{4,5}$ Among various membrane processes, nanofiltration (NF) has particularly attracted more interest thanks to its small pore size yet high flux. ${ }^{6}$ The pore size is defined so that the nonharmful monovalent salts could pass through the membranes, while micropollutants and heavy metals would be easily retained. ${ }^{7}$ The NF process has been successfully applied to numerous separation processes in industrial scales. ${ }^{8-13}$ However, the low stability of NF membranes remains a drawback and often hinders their application in a broader range, where the solution to be filtered can damage the membrane. ${ }^{14,15}$ This is a critical issue because most of the effluents in chemical factories are often at extreme $\mathrm{pH}$ and highly corrosive. ${ }^{16-20}$ Conventional polymer NF membranes quickly lose their performance upon contact with either acidic or alkaline solutions. ${ }^{21}$ Hence, the need for developing highly robust materials for membrane fabrication is rapidly emerging, and this could be achieved by carefully engineering the chemistry of materials.

Thin-film composite (TFC) membranes are specifically of interest for NF processes because of their thin selective layer and robust mechanical strength, leading to a high flux under high pressures. ${ }^{22,23}$ The selective layer in TFC membranes is commonly made through an interfacial polymerization (IP) process, where polymerization occurs at the interface of two immiscible organic and aqueous solutions, each containing a different monomer. ${ }^{24}$ TFC membranes with a polyamide selective layer made via IP between trimesoyl chloride (TMC) and a diamine (e.g., $m$-phenylenediamine, MPD, or piperazine) are the most common form of NF membranes. ${ }^{25,26}$ Although they have shown nearly perfect stability in contact with harsh organic solvents, ${ }^{27,28}$ Lee et al. have reported that polyamide-

Received: September 8, 2021

Accepted: October 18, 2021

Published: October 29, 2021 
based membranes lose their performance upon exposure to a high-pH solution. ${ }^{21,29}$ They proposed that the amide bonds in the polyamide layer are inherently susceptible to hydrolysis under alkaline conditions via direct nucleophilic attack of $\mathrm{OH}^{-}$ to the carbonyl group. Unlike organic solvent nanofiltration processes, aqueous solutions at extreme $\mathrm{pH}$ contain highly reactive ions (i.e., $\mathrm{OH}^{-}$and $\mathrm{H}^{+}$), which interact with the covalent bonds in a polymer chain, rather than the interchain forces (e.g., van der Waals force). ${ }^{30,31}$ Therefore, eliminating the carbonyl groups in the selective layer and producing a polyamine layer instead could substantially enhance the alkaline resistance of the membranes. Given the presence of carbonyl groups in TMC, it is almost impossible to produce an alkaline-resistant TFC membrane when using TMC for IP. ${ }^{21}$ Hence, searching for potential monomers to form a thin polyamine layer through IP is an ongoing research topic.

$1,3,5$-Tris(bromomethyl)benzene (tBrMeB) is a trifunctional monomer with bromo substituents around an aromatic ring that can be attached to amine groups. ${ }^{32}$ It has been previously used as a cross-linking agent to enhance the gas separation performance of polybenzimidazole membranes. ${ }^{32}$ In this work, we investigate the potential of $\mathrm{tBrMeB}$ as the carbonyl free organic phase monomer in an IP reaction. To the best of our knowledge, the IP reaction between $\mathrm{tBrMeB}$ and diamines has never been reported in the open literature. This paper aims to first achieve a freestanding polymer film by using $\mathrm{tBrMeB}$ as the organic phase monomer and PDD as the aqueous phase monomer and then prepare the corresponding polyamine TFC membranes and evaluate their $\mathrm{pH}$ stability. Subsequently, MPD is employed as an alternative monomer to enhance the performance of the TFC membranes. Thorough characterizations would be conducted to understand the chemistry, morphology, and stability of the developed membranes.

\section{EXPERIMENTAL SECTION}

2.1. Materials. $1,3,5$-Tris(bromomethyl)benzene (tBrMeB, $97 \%$ ), p-phenylenediamine (PPD, 99\%), $m$-phenylenediamine (MPD, 99\%), poly(ethylene glycol) (PEG, $M_{\mathrm{w}}=400,600 \mathrm{~g} \mathrm{~mol}^{-1}$ ), sodium chloride $(\mathrm{NaCl}$, ACS reagent, $\geq 99 \%$, anhydrous, Redi-Dri, freeflowing), sodium sulfate $\left(\mathrm{Na}_{2} \mathrm{SO}_{4}\right.$, ACS reagent, $\geq 99 \%$, anhydrous, granular, free-flowing, Redi-DriTM), and sodium hydroxide $(\mathrm{NaOH}$, BioXtra, $\geq 98 \%$ ) were obtained from Sigma-Aldrich. Magnesium sulfate heptahydrate $\left(\mathrm{MgSO}_{4}, \mathrm{ACS}\right.$ reagent. Ph. Eur.), nitric acid $\left(\mathrm{HNO}_{3}, 1 \mathrm{~mol} \mathrm{~L}{ }^{-1}\right.$, Titripur), and PEG 1000 (EMPROVE ESSENTIAL) were supplied by Merck. Magnesium chloride hexahydrate $\left(\mathrm{MgCl}_{2}, 99.0-101.0 \%\right.$ AnalaR NORMAPUR ACS Reag.) was purchased from VWR Chemicals (Netherlands). nHexane (anhydrous, over molecular sieves) was acquired from Alfa Aesar (Germany). PEG 200 was obtained from Fluka (Germany).

Hydrophilized poly(ether sulfone) (PES) ultrafiltration membranes with a molecular weight cutoff (MWCO) of $30 \mathrm{kDa}$ were obtained from Microdyn-Nadir (UH 030, Germany) and were used as support membrane. According to the specifications and a previously reported study, ${ }^{31}$ the PES ultrafiltration support membranes are stable in solutions with $\mathrm{pH}$ ranging from 0 to 14 .

2.2. Synthesis of Freestanding Thin Films. Freestanding polyamine thin films were prepared by interfacial polymerization. PPD was dissolved in water with various concentrations. A solution of $0.7 \mathrm{wt} \% \mathrm{tBrMeB}$ in hexane was slowly added to the aqueous solution and allowed to react for various durations. The reaction duration for each experiment was recorded from the moment of $\mathrm{tBrMeB}$ addition until a visual layer at the interface appeared. Based on the observations, the best conditions were chosen for developing TFC membranes.
2.3. TFC Membrane Preparation. TFC membranes were prepared by IP directly on the PES supports. Prior to IP, PES supports were immersed in water overnight to ensure the complete removal of any surfactants/contaminants. Afterward, IP was performed by first exposing the PES surface to an aqueous solution. In the case of PPD, $1 \mathrm{wt} \%$ PPD in water was exposed to the surface for $2 \mathrm{~min}$. The diamine-loaded PES membranes were then wiped with a tissue paper and passed with a rubber roller to remove excess solution. The membranes were then subjected to a $\mathrm{tBrMeB}$ solution for $30 \mathrm{~min}$. The whole IP setup was encased in a sealed container to prevent hexane evaporation during IP. The resulting TFC membranes were withdrawn from the $\mathrm{tBrMeB}$ solution, rinsed with hexane, dried for $1 \mathrm{~min}$ in air, and then preserved in water for further testing and use. In the case of MPD, 5 wt \% MPD was exposed to the PES surface for $5 \mathrm{~min}$. Then, the solution was drained by applying a vacuum until the surface of the MPD-loaded PES membranes was dry. After this, the membranes were subjected to $\mathrm{tBrMeB}$ for $5 \mathrm{~min}$ and treated as described before.

Fabricated PPD membranes were tested for their reproducibility in terms of $\mathrm{PWP}$ and $\mathrm{NaCl}$ rejection. Seven membrane samples that were prepared under the exact same conditions (i.e., monomer concentration, reaction duration, and volume of the reacting solution) were considered. First, the PWP was measured. Afterward, the pure water was replaced by an aqueous solution containing $1 \mathrm{~g} \mathrm{~L}^{-1} \mathrm{NaCl}$, and the rejection was measured.

2.4. Membrane Characterizations. A field emission scanning electron microscope (FESEM, JSM-7610F) was used to visualize the surface and cross section of the membranes. For the sample preparation, TFC membranes were first immersed in isopropanol 3 times, $30 \mathrm{~min}$ each, followed by soaking 3 times in hexane, $30 \mathrm{~min}$ each. Afterward, the membranes were air-dried, fractured in liquid nitrogen, and coated with an ultrathin $5 \mathrm{~nm} \mathrm{Pt} / \mathrm{Pd}$ layer.

The surface chemistry of the membranes was examined by attenuated total reflectance Fourier transform infrared spectroscopy (ATR-FTIR, PerkinElmer Spectrum Two). Spectra were collected from 16 scans with a resolution of $4 \mathrm{~cm}^{-1}$ over a wavelength range from 400 to $4000 \mathrm{~cm}^{-1}$. The hydrophilicity of the membranes was measured by using a contact angle goniometer in the sessile drop method (DataPhysics OCA20). The error bars in the contact angle results represent the standard deviation of eight measurements.

2.5. Separation Performance. Nanofiltration performance was evaluated by using a dead-end filtration cell at room temperature at 10 bar. The pure water permeance (PWP) was evaluated by using demineralized water and determined according to eq 1 :

$$
\mathrm{PWP}=\frac{V}{A t \Delta P}
$$

Here, PWP is the permeance $\left(\mathrm{L} \mathrm{m}^{-2} \mathrm{~h}^{-1} \mathrm{bar}^{-1}\right), V$ the permeate volume (L), $A$ the membrane area $\left(\mathrm{m}^{2}\right), t$ the filtration time $(\mathrm{h})$, and $\Delta P$ the transmembrane pressure (bar).

The rejection $(R, \%)$ was measured by permeating salt solutions of $1 \mathrm{~g} \mathrm{~L}^{-1}$ containing either $\mathrm{NaCl}, \mathrm{MgCl}_{2}, \mathrm{MgSO}_{4}$, or $\mathrm{Na}_{2} \mathrm{SO}_{4}$. During measurements, the solution was stirred to minimalize concentration polarization. The rejection rate $(R)$ of salts was obtained by using eq 2

$$
R=\left(1-\frac{C_{\mathrm{p}}}{C_{\mathrm{f}}}\right) \times 100 \%
$$

where $C_{\mathrm{p}}$ and $C_{\mathrm{f}}$ correspond to the salt concentrations $\left(\mathrm{mg} \mathrm{L}^{-1}\right)$ in permeate and feed solutions, respectively. A 3310 conductivity meter (WTW, Germany) was employed to analyze the salt concentrations in the feed and the permeate solutions. Error bars depict the standard deviation of either three (PPD) or two (MPD) different membrane samples.

2.5.1. MWCO and Pore Size Distribution. The molecular weight cutoff (MWCO) of the membranes was evaluated with an aqueous solution containing a mixture of PEGs with a molecular weight of 200, 400,600 , and $1000 \mathrm{~g} \mathrm{~mol}^{-1}$ (each fraction $1 \mathrm{~g} \mathrm{~L}^{-1}$ ). Compositions of permeate and feed were analyzed by gel permeation chromatography (GPC, Agilent1200/1260 Infinity GPC/SEC series, Polymer Stand- 
(a)<smiles>CCC1CC(CC)CC(CC)C1</smiles>

1,3,5-Tris(bromomethyl)benzene (tBrMeB )

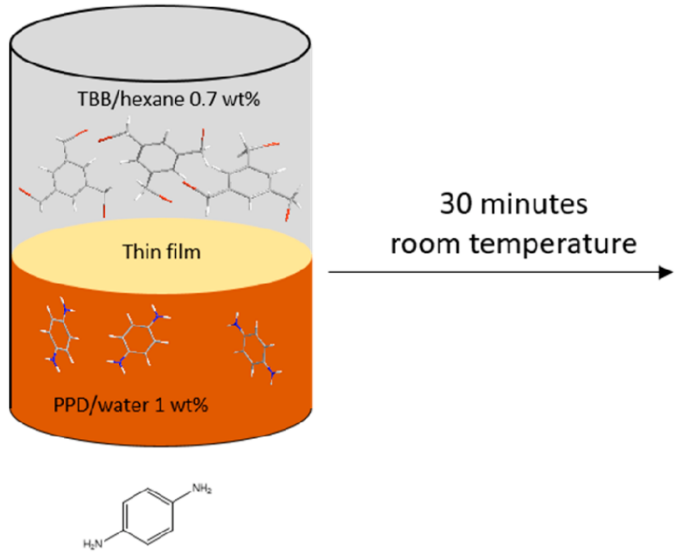

(b)



p-phenylenediamine (PPD)

Figure 1. (a) Visualization of the interfacial polymerization between $t B r M e B$ in hexane and PPD in water. (b) Photograph of a glass vial containing the two immiscible solutions. A thin film is formed between the two phases.

ards Service Suprema $8 \times 300 \mathrm{~mm}$ columns in series $(1000 \AA, 10 \mu \mathrm{M}$; $30 \AA, 10 \mu \mathrm{M})$ ), and the MWCO was obtained from a plot of PEG standards rejection rates versus their molecular weights. The MWCO is defined as the molar mass that is retained for $90 \%$ or more. Error bars depict the standard deviation of either three (PPD) or two (MPD) different membrane samples.

The solute transport experiments were also used to calculate the pore size and the pore size distribution of the membranes. In short, the molecular weight $(M)$ of the PEGs can be related to the solute radius $(r)$ according to eq 3 .

$$
r=16.73 \times 10^{-12} M^{0.557}
$$

When the solute rejection $(R)$ is plotted against the solute radius $(r)$ on a log-normal probability graph, a linear relationship between $R$ and $r$ can be observed. The mean effective pore size $\left(\mu_{\mathrm{p}}\right)$ can then be obtained by assuming it to be the same as $r$ when $R=50 \%$.

Finally, the pore size distribution can be obtained by using the probability density function in eq 4 .

$$
\frac{\mathrm{d} R\left(d_{\mathrm{p}}\right)}{\mathrm{d} d_{\mathrm{p}}}=\frac{1}{d_{\mathrm{p}} \ln \sigma_{\mathrm{p}} \sqrt{2 \pi}} \exp \left[-\frac{\left(\ln d_{\mathrm{p}}-\ln \mu_{\mathrm{p}}\right)^{2}}{2\left(\ln \sigma_{\mathrm{p}}\right)^{2}}\right]
$$

where $d_{\mathrm{p}}$ is the effective pore diameter and $\sigma_{\mathrm{p}}$ is the geometric standard, defined as the ratio between the values of $r$ at $R=84.13 \%$ and $R=50 \%$. Further details can be found in ref 33 .

2.6. pH Stability. To determine the robustness of the membranes in contact with solutions at extreme $\mathrm{pH}$, two sets of experiments were designed and conducted.

2.6.1. Ex Situ $p H$ Stability. First, the $\mathrm{pH}$ stability was measured ex situ. This means that after measuring the nanofiltration performance, the membrane samples were stored in an aqueous solution of either $0.1 \mathrm{M} \mathrm{NaOH}$ or $0.1 \mathrm{M} \mathrm{HNO}_{3}$ for regular time intervals at room temperature. After the exposure, the membrane samples were thoroughly washed with DI water, and their nanofiltration performance was evaluated. Error bars depict the standard deviation of either three (PPD) or two (MPD) different membrane samples.

2.6.2. In Situ pH Stability. In the in situ experiments, the PWP and the salt rejection of the pristine membranes were measured, followed by filtration of $0.1 \mathrm{M} \mathrm{NaOH}$ solution for $6 \mathrm{~h}$. Afterward, the nanofiltration performance was measured again. Next, a solution of $0.1 \mathrm{M} \mathrm{HNO}_{3}$ was filtered through the membrane for $6 \mathrm{~h}$. Finally, the nanofiltration performance of the membrane was measured for the last time.

\section{RESULTS AND DISCUSSION}

3.1. Freestanding Thin Films. We chose the interfacial polymerization method because it produces highly cross-linked and thin films, which are needed to achieve high selectivity and stability in harsh environments. This facile method has been extensively used to synthesize polyamide-based membranes from the reaction between TMC and diamines (e.g., MPD). However, to the best of our knowledge, the interfacial polymerization of $\mathrm{tBrMeB}$ with amine monomers has not been reported. Therefore, the optimized conditions to conduct interfacial polymerization reaction between $\mathrm{tBrMeB}$ and PPD will be first presented.

The optimum conditions for the interfacial polymerization reaction, resulting in an interconnected firm layer, are illustrated in Figure 1. In short, 1 wt \% PPD was allowed to react with 0.7 wt $\% \mathrm{tBrMeB}$ for $30 \mathrm{~min}$ at room temperature.

3.2. Interfacial Polymerization on PES Support. The optimum conditions for interfacial polymerization were used to prepare TFC membranes with PPD as the aqueous phase and tBrMeB as the organic phase monomers. To evaluate the success of IP on the PES support, the NF performance of the TFC membranes and that of PES supports were measured and are reported in Table 1 and Figure 2.

The dramatic reduction of MWCO and the PWP suggests the presence of a dense layer atop of the PES support. These results are consistent with the morphological observations in Figure 3. Unlike the porous surface of PES with visible pores, the surface of TFC membrane is rather dense. However, the

Table 1. TFC Membrane Properties

\begin{tabular}{cccc}
\multicolumn{1}{c}{ property } & $\begin{array}{c}\text { PES } \\
\text { support }\end{array}$ & $\begin{array}{c}\text { PPD-tBrMeB } \\
\text { membrane }\end{array}$ & $\begin{array}{c}\text { MPD-tBrMeB } \\
\text { membrane }\end{array}$ \\
$\begin{array}{c}\text { PWP }\left(\mathrm{L} \mathrm{m}^{-2} \mathrm{~h}^{-1}\right. \\
\left.\mathrm{bar}^{-1}\right)\end{array}$ & $51 \pm 5.3$ & $0.28 \pm 0.09$ & $1.3 \pm 0.3$ \\
$\begin{array}{l}\mathrm{MgSO}_{4} \text { rejection } \\
(\%)\end{array}$ & $\mathrm{n} / \mathrm{a}$ & $68 \pm 3.9$ & $67 \pm 1.2$ \\
$\mathrm{MWCO}\left(\mathrm{g} \mathrm{mol}^{-1}\right)$ & $30000^{a}$ & $820 \pm 132$ & $566 \pm 43$
\end{tabular}

${ }^{a}$ According to the supplier. 

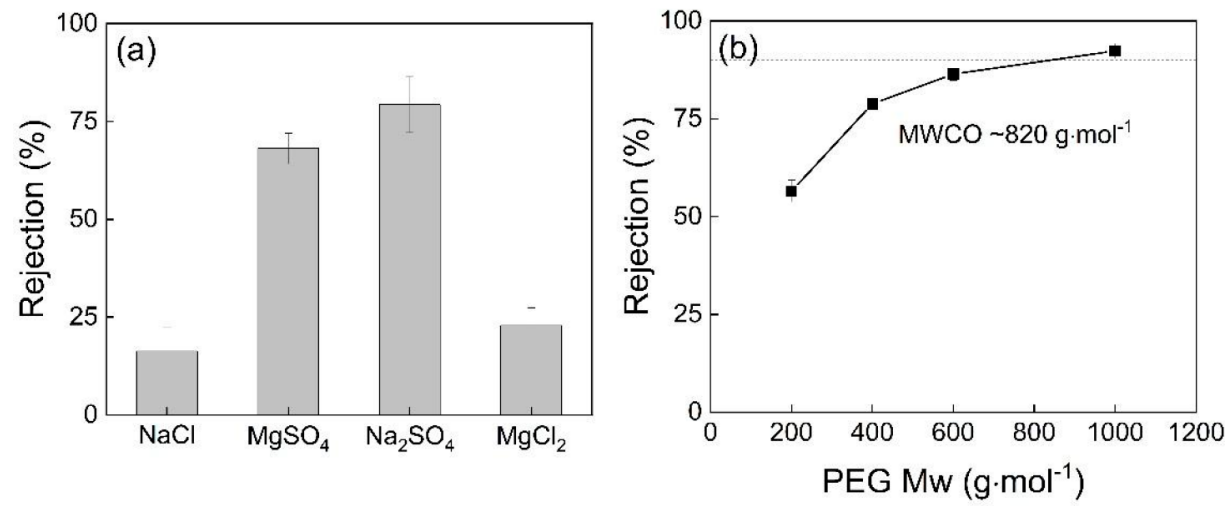

Figure 2. PPD-tBrMeB membranes: (a) salt rejection and (b) PEG rejection.
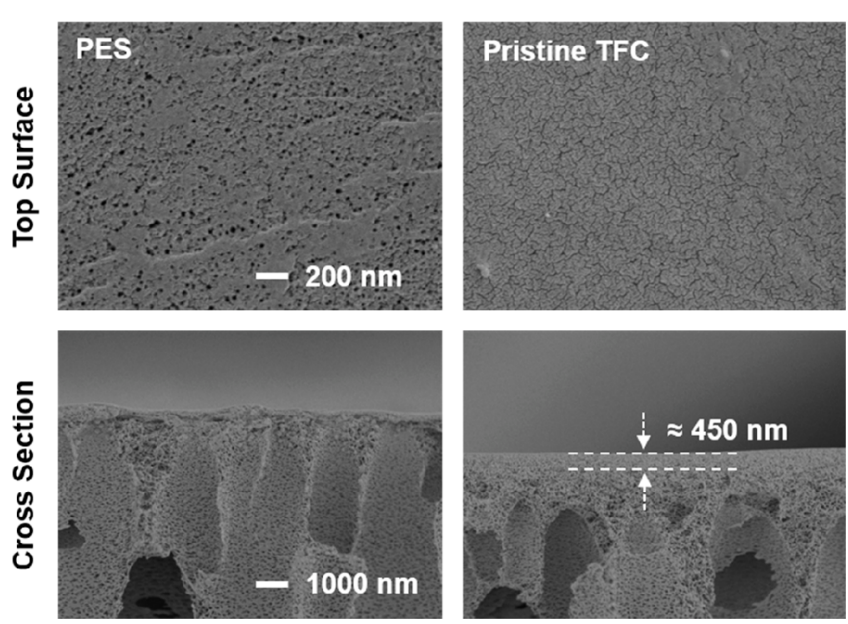

Figure 3. Morphology of the PES support and TFC PPD-tBrMeB membrane.

surface morphology of the TFC membrane differs from the socalled ridge and valley structure of the polyamide TFC membranes. The slow reaction rate of $\mathrm{tBrMeB}$ could be the cause for this discrepancy. As the interfacial polymerization progresses, the newly formed polymers have enough time to rearrange and pack in a more ordered manner. The thickness of the IP layer is roughly $450 \mathrm{~nm}$.

Figure 4 compares the FTIR spectra of the PES and the TFC membrane. The characteristic peaks of PES are consistent with the PES spectra published in the literature. ${ }^{21} \mathrm{~A}$

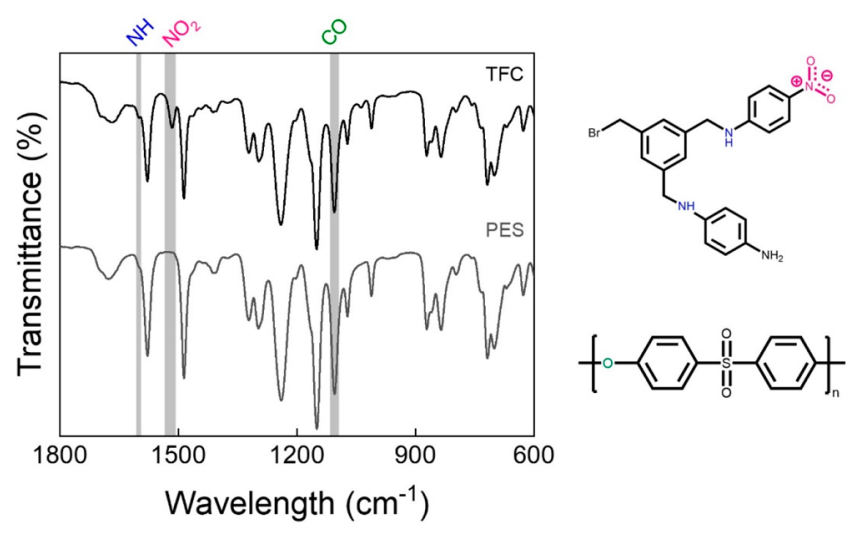

Figure 4. Comparison between the FTIR spectra of the PES support and PPD-tBrMeB membrane. distinguishable peak at $1514 \mathrm{~cm}^{-1}$ appears after thin film deposition on the PES support which can be assigned to $\mathrm{NO}_{2}$ (the oxidized form of the unreacted terminal of PPD). The peak at $1600 \mathrm{~cm}^{-1}$ may be attributed to the secondary amine that is expected in the tBrMeB-PPD network.

3.3. Reproducibility of the Membranes. The results of the reproducibility measurements are depicted in the Supporting Information (Figure S1). Although the variation of rejection is almost negligible, PWP shows some discrepancy. This scattering could be due to the noncontrollable parameters of the interfacial polymerization process, such as uniformity of the porosity, draining of excessive reacting solutions, and so on. Nevertheless, the individual deviation for both permeance and rejection is $<10 \%$.

3.4. pH Stability. 3.4.1. Ex Situ Filtration. Figure 5 shows the evolution of PWP and $\mathrm{MgCl}_{2}$ rejection after prolonged exposure, up to 2 months, to acidic and alkaline conditions. Both permeance and rejection show an initial rapid increase, which then levels off to a constant value. Under acidic conditions, the permeance increases from $0.3 \pm 0.07$ to $1.27 \pm$ $0.09 \mathrm{~L} \mathrm{~m}^{-2} \mathrm{~h}^{-1} \mathrm{bar}^{-1}$ and the rejection from $21 \pm 4$ to $52 \pm$ $3 \%$. Under alkaline conditions, the increase is less severe, the permeance increases from $0.25 \pm 0.04$ to $0.46 \pm 0.04 \mathrm{~L} \mathrm{~m}^{-2}$ $\mathrm{h}^{-1} \mathrm{bar}^{-1}$, and the rejection increases from $21 \pm 2$ to $27 \pm 2 \%$. The initial performance increase can be explained by the chemical cleaning effects of acidic/alkaline solutions, where the low molecular weight polymers or/and unreacted monomers are washed away, making water to pass through more easily, resulting in a higher salt rejection. Additionally, nitric acid tends to form hydrogen bonds with amine groups, resulting in a tighter pore size. ${ }^{34}$ Nevertheless, the results show that the TFC membranes are stable in contact with extreme $\mathrm{pH}$ for a prolonged time.

3.4.2. In Situ Filtration. To better demonstrate the robustness of the membranes and their applications for filtration under extreme $\mathrm{pH}$ conditions, an in situ exposure experiment was carried out. Both acidic and alkaline solutions were filtered through the membrane. As can be seen in Figure $6 \mathrm{a}$, the membrane performance after the second cycle (i.e., alkaline filtration) remains almost unchanged, while after acidic filtration, all the salt rejections increase. This observation is in good agreement with the results from ex situ filtration experiments where exposure to acidic solutions had a more severe effect than to the alkaline one. Figures $6 \mathrm{~b}$ and $6 \mathrm{c}$ show the MWCO and the pore size distribution of the membrane after each cycle, respectively. It is evident that the acidic filtration reduces the membrane pore size and narrows down 

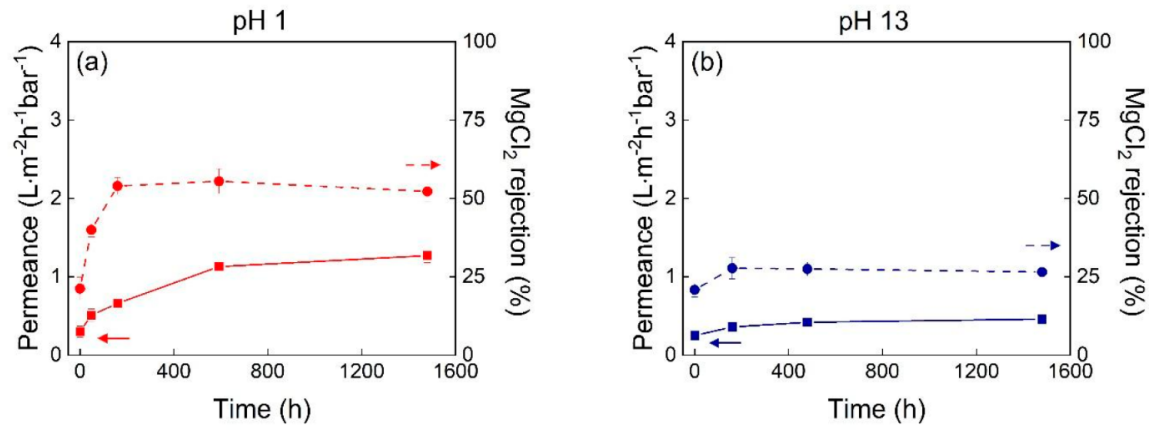

Figure 5. Long-term $\mathrm{pH}$ stability tests of PPD-tBrMeB membranes after prolonged treatment in (a) $0.1 \mathrm{M} \mathrm{HNO}_{3}$ (red) and (b) $0.1 \mathrm{M} \mathrm{NaOH}$ solutions (blue). Water permeance on left $y$-axis (squares, solid line) and $\mathrm{MgCl}_{2}$ rejection on right $y$-axis (circles, dashed line).
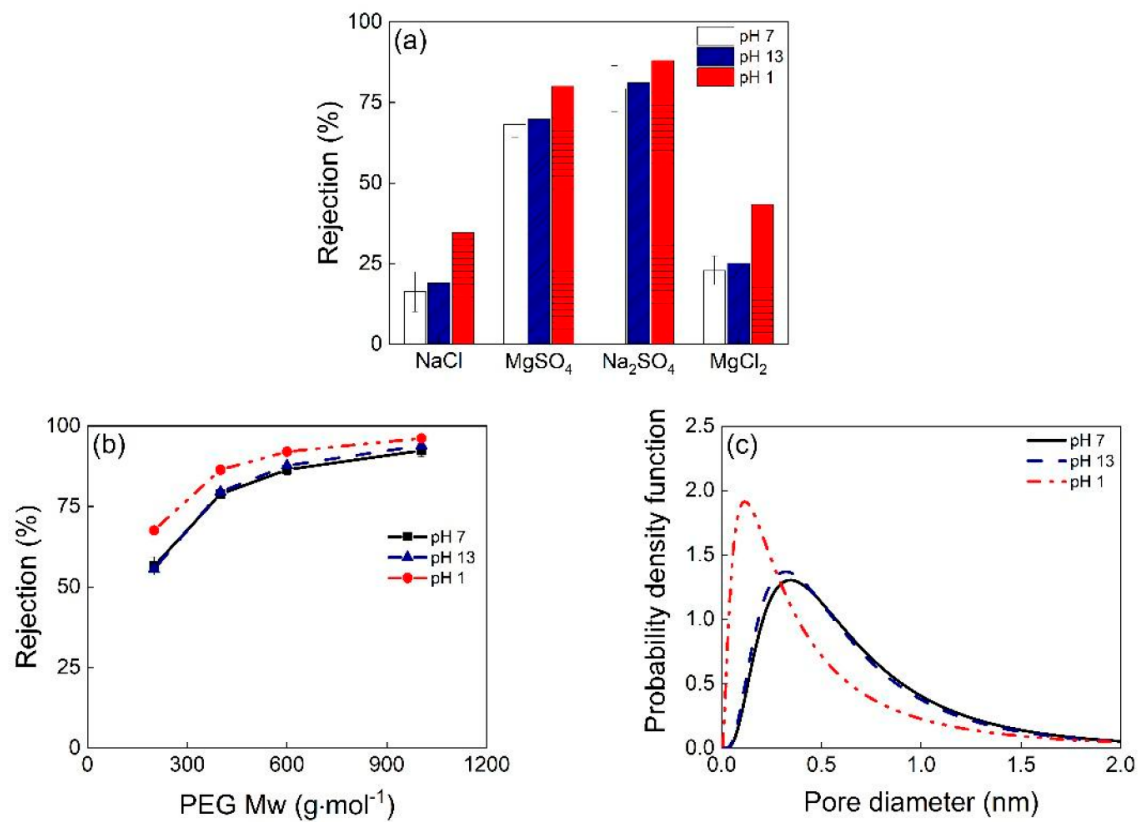

Figure 6. (a) Salt rejection, (b) PEG rejection, and (c) pore size distribution of the tBrMeB-PPD membrane before and after in situ exposure to 0.1 $\mathrm{M} \mathrm{NaOH}$ and $0.1 \mathrm{M} \mathrm{HNO}_{3}$.

its distribution. The MWCO reduces from 820 to $480 \mathrm{~g} \mathrm{~mol}^{-1}$ after acidic filtration, for which the origin has been explained in the previous paragraph.

3.4.3. Morphology and Surface Analysis. It has been shown that the membrane performance of PPD-tBrMeB membranes is stable after extreme $\mathrm{pH}$ exposure. Next to the performance, we have also analyzed the morphology and surface changes of the membranes before and after $\mathrm{pH}$ exposure. Figure 7 shows the FESEM images of the surface, cross section, and selective layer of the membranes. When comparing the images of the membranes before and after $\mathrm{pH}$ exposure, we could find no clear difference; for example, the selective layer is intact, which is consistent with the other results.

In Figure 8 the water contact angle of the PES support and TFC membranes before and after $\mathrm{pH}$ exposure is shown. It shows that the contact angle after $\mathrm{pH} 13$ is similar to the pristine membrane $\left(70^{\circ}\right)$, while after $\mathrm{pH} 1$ the contact angle drops $\left(57^{\circ}\right)$. This indicates that the membrane becomes more hydrophilic after exposure to $\mathrm{pH} 1$ and might also explain the higher PWP.

3.4.4. Surface Chemistry. To further confirm the stability of the membranes in extreme $\mathrm{pH}$ conditions, the surface chemistry of the membranes before and after $\mathrm{pH}$ treatment was studied by means of FTIR. From Figure S2 it can be concluded that there is no clear change in the chemistry of the membranes after $\mathrm{pH}$ exposure as the FTIR spectra are the same. This substantiates that the amine bonds are stable under the exposed conditions. Nevertheless, a more sensitive chemistry analysis method would be needed to confirm that the chemistry of the membranes really remains intact.

3.5. Enhanced Performance. We have shown the successful preparation of PPD-tBrMeB TFC membranes with great $\mathrm{pH}$ stability. However, the permeance of these membranes can be considered rather low. To enhance the performance of the TFC membranes, $m$-phenylenediamine (MPD) was used as an alternative monomer to PPD. Earlier research has shown that MPD has a lower reactivity than PPD and in general leads to a rougher surface that increases the membrane surface area, a better hydrophilicity, and a higher water flux. ${ }^{35,36}$

Therefore, TFC membranes were also prepared via IP with 5 wt $\% \mathrm{MPD}$ in water as the aqueous phase and 0.7 wt \% $\mathrm{tBrMeB}$ in hexane as the organic phase and allowed to react for $5 \mathrm{~min}$. These conditions were chosen such that the $\mathrm{MgSO}_{4}$ rejection was comparable to that of PPD membranes, and the 

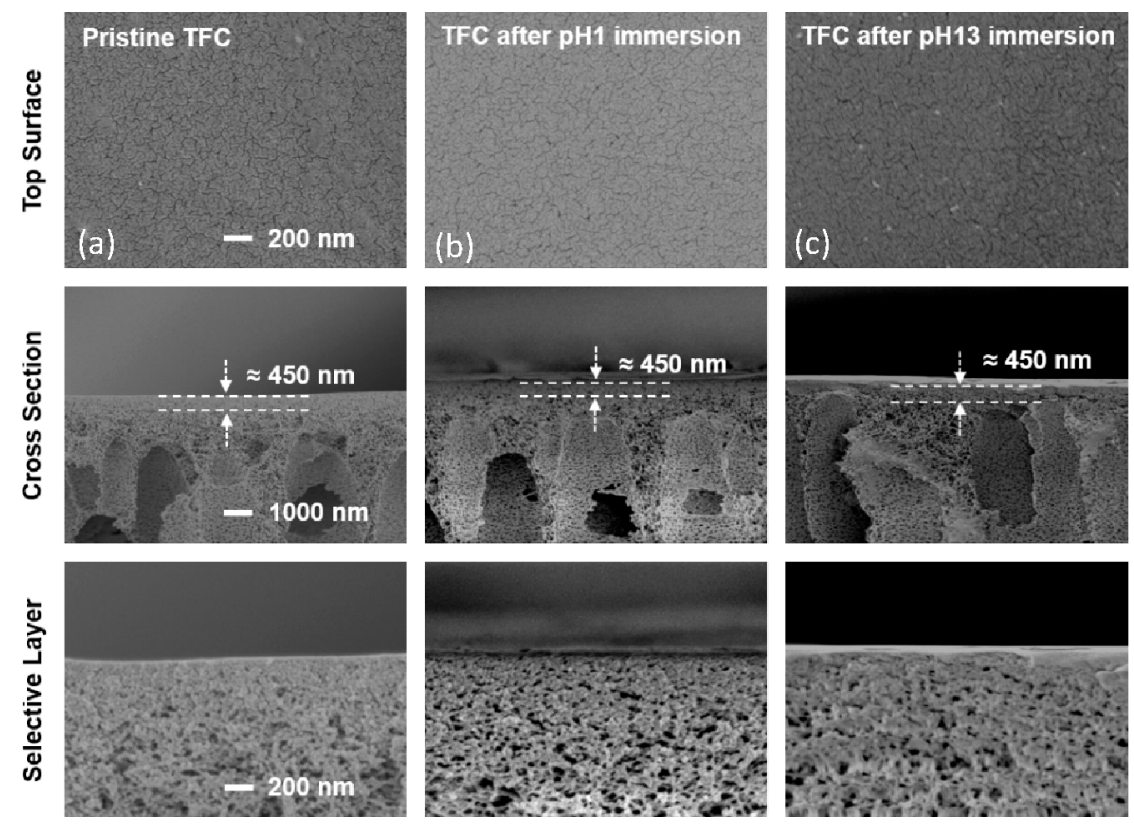

Figure 7. Morphology of the (a) pristine PPD-tBrMeB membrane, (b) after 4 weeks immersion in $0.1 \mathrm{M} \mathrm{NaOH}$, and (c) after 4 weeks immersion in $0.1 \mathrm{M} \mathrm{HNO}_{3}$.

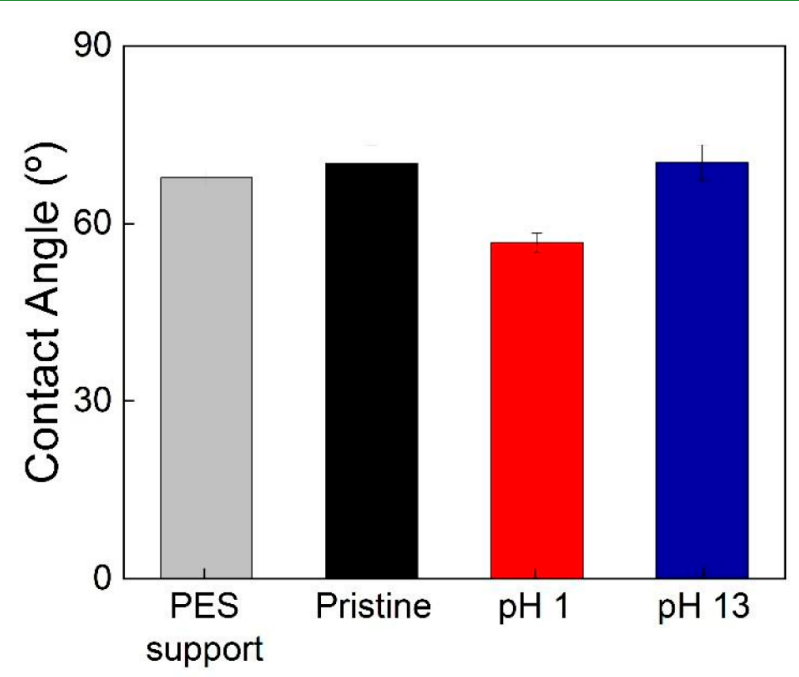

Figure 8. Water contact angle of PES support, pristine, and after prolonged immersion in $0.1 \mathrm{M} \mathrm{NaOH}$ or $0.1 \mathrm{M} \mathrm{HNO}_{3}$ TFC membranes.

performance in terms of PWP and MWCO could be evaluated. The resulting MPD-tBrMeB membranes show a permeance of $1.3 \pm 0.3 \mathrm{~L} \mathrm{~m}^{-2} \mathrm{~h}^{-1} \mathrm{bar}^{-1}$ and have a MWCO of $566 \pm 43 \mathrm{~g}$ $\mathrm{mol}^{-1}$ (Table 1 and Figure S3). Compared to the pristine values of membranes prepared with PPD, this indicates 3 times higher permeance at similar rejection values and a lower MWCO. Therefore, it can be concluded that the use of MPD as a monomer enhances the performance of this type of membrane. The more hydrophilic character of membranes prepared with MPD is also confirmed by the contact angle data (Figure S4), showing a slightly lower value than for PPD membranes $\left(63^{\circ}\right.$ vs $\left.70^{\circ}\right)$.

Figure S5 shows FESEM images of the MPD-tBrMeB membranes. Compared to the PPD membranes, it shows a rougher surface with a kind of nodular structure. The IP layer has a thickness of $\sim 640 \mathrm{~nm}$, which is thicker than the PPD layer. Therefore, the increased permeance is not a result of a thinner layer and lower resistance but probably because of the more hydrophilic structure (Figure S4). Moreover, TFC membranes made of MPD tend to have higher free volume as compared to PPD.

To be certain about the $\mathrm{pH}$ stability, also here ex situ $\mathrm{pH}$ stability tests were performed, and the results are shown in Figure 9. Similar to the results obtained with PPD, the membranes show stable performance after exposure to $\mathrm{pH}$ solutions for a prolonged time. After acidic exposure, an increase in salt rejection is observed from $62.0 \pm 9.3$ to $84.9 \pm$ $2.6 \%$, which can also be explained by the hydrogen bond formation between the selective layer and nitric acid.

Overall, it can be concluded that amine-tBrMeB NF membranes show excellent long-term $\mathrm{pH}$ stability and therefore have a great potential for use under these extreme conditions. By tuning the monomers, for example, by using MPD instead of PPD, we can improve the performance of the membranes.

\section{CONCLUSIONS}

This study shows the fabrication of a robust membrane by interfacial polymerization of $\mathrm{tBrMeB}$ with the amines PPD and MPD that can withstand prolonged exposure to extreme $\mathrm{pH}$ conditions. PPD-tBrMeB thin-film composite membranes show performances in the NF range, with a clean water permeability of $0.28 \pm 0.09 \mathrm{~L} \mathrm{~m}^{-2} \mathrm{~h}^{-1} \mathrm{bar}^{-1}$, a $68 \pm 3.9 \%$ $\mathrm{MgSO}_{4}$ rejection, and a MWCO of $820 \pm 132 \mathrm{~g} \mathrm{~mol}^{-1}$. Both ex situ and in situ $\mathrm{pH}$ stability tests prove their long-term (up to 2 months) $\mathrm{pH}$ stability under extreme $\mathrm{pH}$ conditions. Furthermore, the performance could be enhanced by using MPD instead of PPD as the aqueous phase monomer, resulting in an increased permeance from $0.28 \pm 0.09$ to $1.3 \pm 0.3 \mathrm{~L}$ $\mathrm{m}^{-2} \mathrm{~h}^{-1}$ bar $^{-1}$, while keeping similar salt rejections and lowering the MWCO. These membranes have the potential to be used as an alternative to conventional TFC membranes and treat wastewater streams at extreme $\mathrm{pH}$ conditions. 

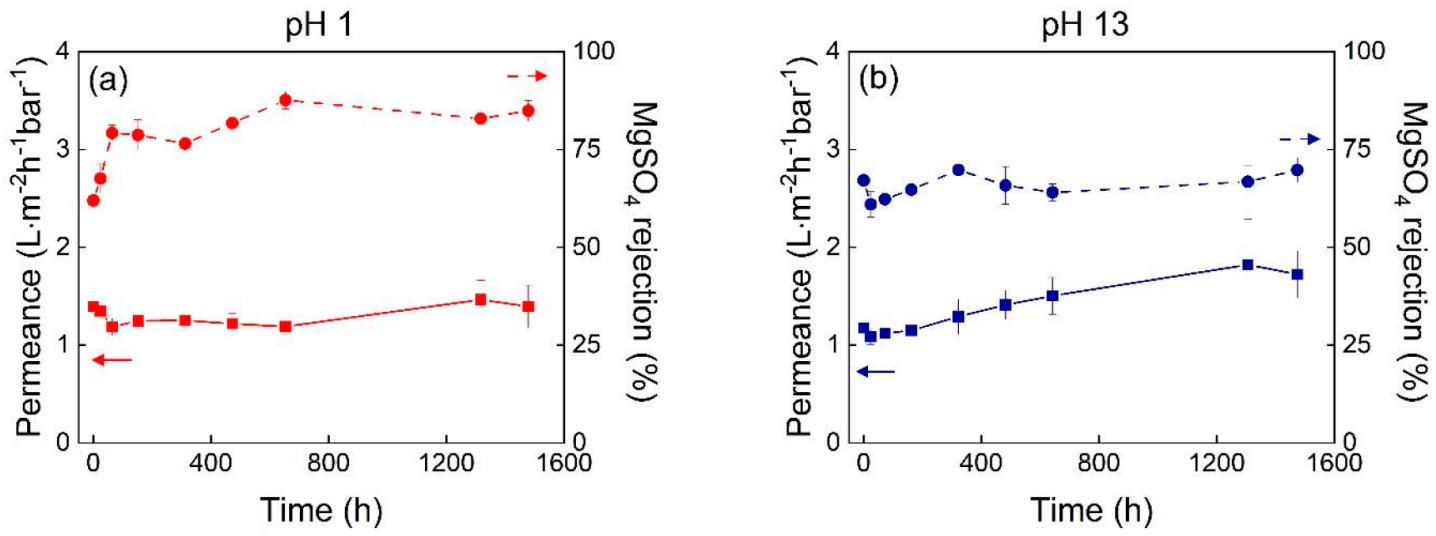

Figure 9. Long-term $\mathrm{pH}$ stability tests of $\mathrm{MPD}-\mathrm{tBrMeB}$ membranes after prolonged treatment in (a) $0.1 \mathrm{M} \mathrm{HNO}_{3}$ (red) and (b) $0.1 \mathrm{M} \mathrm{NaOH}$ solutions (blue). Water permeance on the left $y$-axis (squares, solid line) and $\mathrm{MgSO}_{4}$ rejection on the right $y$-axis (circles, dashed line).

\section{ASSOCIATED CONTENT}

\section{SI Supporting Information}

The Supporting Information is available free of charge at https://pubs.acs.org/doi/10.1021/acsapm.1c01172.

Figure S1, demonstrating the reproducibility of the interfacial polymerization; Figure S2, showing the FTIR spectra before and after $\mathrm{pH}$ treatment; Figure S3, demonstrating the MWCO of MPD-tBrMeB membrane; Figure S4, depicting the water contact angle of the PES support and TFC membranes; Figure S5, showing the morphology of MPD-tBrMeB membranes; Table S1, listing the various conditions tested for IP reaction; Table S2, comparing the performance of the developed membranes with the commercially available membranes (PDF)

\section{AUTHOR INFORMATION}

\section{Corresponding Authors}

Akbar Asadi Tashvigh - Biobased Chemistry and Technology, Wageningen University \& Research, 6708 WG Wageningen, The Netherlands; 이이.org/0000-0003-0454-0314; Phone: (+31)317480694; Email: akbar.asaditashvigh@ wur.nl

Nieck E. Benes - Films in Fluids Group, Membrane Science and Technology Cluster, Faculty of Science and Technology, MESA+ Institute for Nanotechnology, University of Twente, 7500 AE Enschede, The Netherlands; (1) orcid.org/00000001-9716-069X; Phone: (+31)534894288;

Email: n.e.benes@utwente.nl

\section{Author}

Maria G. Elshof - Films in Fluids Group, Membrane Science and Technology Cluster, Faculty of Science and Technology, MESA+ Institute for Nanotechnology, University of Twente, 7500 AE Enschede, The Netherlands; (1) orcid.org/00000002-4038-1413

Complete contact information is available at:

https://pubs.acs.org/10.1021/acsapm.1c01172

\section{Author Contributions}

A.A.T. contributed to conceptualization, experimental design, methodology, and preparation of the first draft of the manuscript. M.G.E. contributed to experimental design, characterization, discussion, and preparation of the first draft of the manuscript. N.E.B. contributed to conceptualization and provided helpful feedback and discussions on the manuscript.

\section{Notes}

The authors declare no competing financial interest.

\section{ACKNOWLEDGMENTS}

This work was funded by the Dutch Research Council (NWO).

\section{REFERENCES}

(1) Pickering, A. J.; Davis, J. Freshwater availability and water fetching distance affect child health in sub-Saharan Africa. Environ. Sci. Technol. 2012, 46, 2391-2397.

(2) Eggen, R. I. L.; Hollender, J.; Joss, A.; Schärer, M.; Stamm, C. Reducing the Discharge of Micropollutants in the Aquatic Environment: The Benefits of Upgrading Wastewater Treatment Plants. Environ. Sci. Technol. 2014, 48, 7683-7689.

(3) Mekonnen, M. M.; Hoekstra, A. Y. Global gray water footprint and water pollution levels related to anthropogenic nitrogen loads to fresh water. Environ. Sci. Technol. 2015, 49, 12860-12868.

(4) Tang, C. Y.; Yang, Z.; Guo, H.; Wen, J. J.; Nghiem, L. D.; Cornelissen, E. Potable Water Reuse through Advanced Membrane Technology. Environ. Sci. Technol. 2018, 52, 10215-10223.

(5) Van der Bruggen, B.; Vandecasteele, C. Distillation vs. membrane filtration: overview of process evolutions in seawater desalination. Desalination 2002, 143, 207-218.

(6) McGinnis, R. L.; Reimund, K.; Ren, J.; Xia, L.; Chowdhury, M. R.; Sun, X.; Abril, M.; Moon, J. D.; Merrick, M. M.; Park, J.; Stevens, K. A.; McCutcheon, J. R.; Freeman, B. D. Large-scale polymeric carbon nanotube membranes with sub-1.27-nm pores. Sci. Adv. 2018, 4, e1700938.

(7) Mohammad, A. W.; Teow, Y.; Ang, W.; Chung, Y.; OatleyRadcliffe, D.; Hilal, N. Nanofiltration membranes review: Recent advances and future prospects. Desalination 2015, 356, 226-254.

(8) Remmen, K.; Schäfer, R.; Hedwig, S.; Wintgens, T.; Wessling, M.; Lenz, M. Layer-by-layer membrane modification allows scandium recovery by nanofiltration. Environ. Sci. Water Res. Technol. 2019, 5, 1683-1688

(9) Rasool, M. A.; Van Goethem, C.; Vankelecom, I. F. Green preparation process using methyl lactate for cellulose-acetate-based nanofiltration membranes. Sep. Purif. Technol. 2020, 232, 115903.

(10) Xia, L.; McCutcheon, J. R. Understanding the influence of solvents on the intrinsic properties and performance of polyamide thin film composite membranes. Sep. Purif. Technol. 2020, 238, 116398.

(11) Asadi Tashvigh, A.; Feng, Y.; Weber, M.; Maletzko, C.; Chung, T.-S. 110th Anniversary: Selection of Cross-Linkers and CrossLinking Procedures for the Fabrication of Solvent-Resistant Nano- 
filtration Membranes: A Review. Ind. Eng. Chem. Res. 2019, 58, 10678-10691.

(12) Schnoor, J.-K.; Fuchs, M.; Böcking, A.; Wessling, M.; Liauw, M. A. Homogeneous Catalyst Recycling and Separation of a Multicomponent Mixture Using Organic Solvent Nanofiltration. Chem. Eng. Technol. 2019, 42, 2187-2194.

(13) Aristizábal, S. L.; Chisca, S.; Pulido, B. A.; Nunes, S. P. Preparation of PEEK Membranes with Excellent Stability Using Common Organic Solvents. Ind. Eng. Chem. Res. 2020, 59, 52185226.

(14) Ducom, G.; Cabassud, C. Interests and limitations of nanofiltration for the removal of volatile organic compounds in drinking water production. Desalination 1999, 124, 115-123.

(15) Remmen, K.; Müller, B.; Köser, J.; Wessling, M.; Wintgens, T. Assessment of Layer-By-Layer Modified Nanofiltration Membrane Stability in Phosphoric Acid. Membranes 2020, 10, 61.

(16) Van Goethem, C.; Mertens, M.; Vankelecom, I. F. Crosslinked PVDF membranes for aqueous and extreme $\mathrm{pH}$ nanofiltration. $J$. Membr. Sci. 2019, 572, 489-495.

(17) Trägårdh, G.; Johansson, D. Purification of alkaline cleaning solutions from the dairy industry using membrane separation technology. Desalination 1998, 119, 21-29.

(18) Bargeman, G.; Steensma, M.; ten Kate, A.; Westerink, J.; Demmer, R.; Bakkenes, H.; Manuhutu, C. Nanofiltration as energyefficient solution for sulfate waste in vacuum salt production. Desalination 2009, 245, 460-468.

(19) Jakobs, D.; Baumgarten, G. Nanofiltration of nitric acidic solutions from picture tube production. Desalination 2002, 145, 6568 .

(20) Blöcher, C.; Niewersch, C.; Melin, T. Phosphorus recovery from sewage sludge with a hybrid process of low pressure wet oxidation and nanofiltration. Water Res. 2012, 46, 2009-2019.

(21) Lee, K. P.; Zheng, J.; Bargeman, G.; Kemperman, A. J.; Benes, N. E. pH stable thin film composite polyamine nanofiltration membranes by interfacial polymerisation. J. Membr. Sci. 2015, 478, $75-84$.

(22) Asadi Tashvigh, A.; Chung, T.-S. Facile fabrication of solvent resistant thin film composite membranes by interfacial crosslinking reaction between polyethylenimine and dibromo-p-xylene on polybenzimidazole substrates. J. Membr. Sci. 2018, 560, 115-124.

(23) Lau, W.; Ismail, A.; Misdan, N.; Kassim, M. A recent progress in thin film composite membrane: a review. Desalination 2012, 287, 190-199.

(24) Ismail, A.; Padaki, M.; Hilal, N.; Matsuura, T.; Lau, W. Thin film composite membrane-Recent development and future potential. Desalination 2015, 356, 140-148.

(25) Bui, N.-N.; Lind, M. L.; Hoek, E. M.; McCutcheon, J. R. Electrospun nanofiber supported thin film composite membranes for engineered osmosis. J. Membr. Sci. 2011, 385, 10-19.

(26) Verbeke, R.; Bergmaier, A.; Eschbaumer, S.; Gómez, V.; Dollinger, G. n.; Vankelecom, I. Elemental Depth Profiling of Chlorinated Polyamide-Based Thin-Film Composite Membranes with Elastic Recoil Detection. Environ. Sci. Technol. 2019, 53, 8640-8648.

(27) Hermans, S.; Dom, E.; Mariën, H.; Koeckelberghs, G.; Vankelecom, I. F. Efficient synthesis of interfacially polymerized membranes for solvent resistant nanofiltration. J. Membr. Sci. 2015, 476, 356-363.

(28) Hermans, S.; Mariën, H.; Van Goethem, C.; Vankelecom, I. F. Recent developments in thin film (nano) composite membranes for solvent resistant nanofiltration. Curr. Opin. Chem. Eng. 2015, 8, 4554.

(29) Lee, K. P.; Bargeman, G.; de Rooij, R.; Kemperman, A. J.; Benes, N. E. Interfacial polymerization of cyanuric chloride and monomeric amines: $\mathrm{pH}$ resistant thin film composite polyamine nanofiltration membranes. J. Membr. Sci. 2017, 523, 487-496.

(30) Yao, C.; Burford, R.; Fane, A.; Fell, C.; McDonogh, R. Hydrolysis and other phenomena affecting structure and performance of polyamide 6 membrane. J. Appl. Polym. Sci. 1987, 34, 2399-2408.
(31) Dalwani, M.; Benes, N. E.; Bargeman, G.; Stamatialis, D.; Wessling, M. Effect of $\mathrm{pH}$ on the performance of polyamide/ polyacrylonitrile based thin film composite membranes. J. Membr. Sci. 2011, 372, 228-238.

(32) Naderi, A.; Asadi Tashvigh, A.; Chung, T.-S. $\mathrm{H}_{2} / \mathrm{CO}_{2}$ separation enhancement via chemical modification of polybenzimidazole nanostructure. J. Membr. Sci. 2019, 572, 343-349.

(33) Youm, K. H.; Kim, W. S. Prediction of intrinsic pore properties of ultrafiltration membrane by solute rejection curves: effects of operating conditions on pore properties. J. Chem. Eng. Jpn. 1991, 24, $1-7$.

(34) Asadi Tashvigh, A.; Chung, T.-S. Robust polybenzimidazole (PBI) hollow fiber membranes for organic solvent nanofiltration. J. Membr. Sci. 2019, 572, 580-587.

(35) Kwak, S. Y.; Jung, S. G.; Yoon, Y. S.; Ihm, D. W. Details of surface features in aromatic polyamide reverse osmosis membranes characterized by scanning electron and atomic force microscopy. $J$. Polym. Sci., Part B: Polym. Phys. 1999, 37, 1429-1440.

(36) Juhn Roh, I. Effect of the physicochemical properties on the permeation performance in fully aromatic crosslinked polyamide thin films. J. Appl. Polym. Sci. 2003, 87, 569-576. 